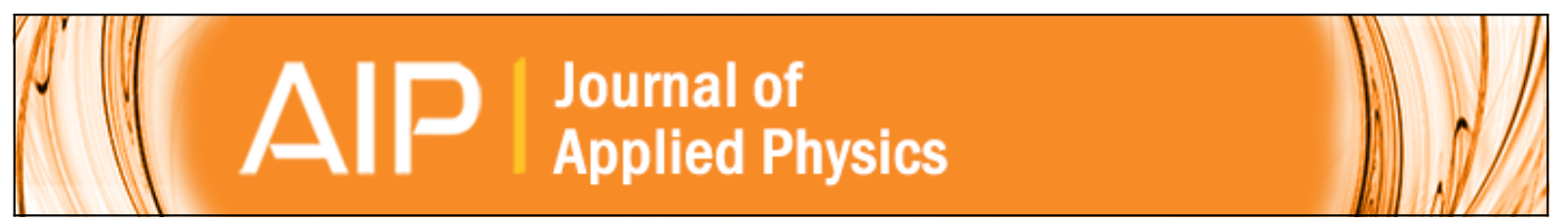

\title{
Perpendicular magnetic anisotropy in epitaxially strained cobalt-ferrite (001) thin films
}

H. Yanagihara, Y. Utsumi, T. Niizeki, J. Inoue, and Eiji Kita

Citation: Journal of Applied Physics 115, 17 A719 (2014); doi: 10.1063/1.4864048

View online: http://dx.doi.org/10.1063/1.4864048

View Table of Contents: http://scitation.aip.org/content/aip/journal/jap/115/17?ver=pdfcov

Published by the AIP Publishing

\section{Articles you may be interested in}

Anomalous thickness-dependent strain states and strain-tunable magnetization in Zn-doped ferrite epitaxial films J. Appl. Phys. 115, 173505 (2014); 10.1063/1.4874920

Erratum: "Extraordinarily large perpendicular magnetic anisotropy in epitaxially strained cobalt-ferrite CoxFe3xO4(001) (x=0.75, 1.0) thin films" [Appl. Phys. Lett. 103, 162407 (2013)]

Appl. Phys. Lett. 104, 059902 (2014); 10.1063/1.4864102

Electron theory of perpendicular magnetic anisotropy of Co-ferrite thin films

AIP Advances 4, 027111 (2014); 10.1063/1.4866279

Extraordinarily large perpendicular magnetic anisotropy in epitaxially strained cobalt-ferrite CoxFe3xO4(001) ( $\mathrm{x}=$ $0.75,1.0)$ thin films

Appl. Phys. Lett. 103, 162407 (2013); 10.1063/1.4824761

Perpendicular magnetic anisotropy in $\mathrm{CoFe} 2 \mathrm{O} 4(001)$ films epitaxially grown on $\mathrm{MgO}(001)$

J. Appl. Phys. 109, 07C122 (2011); 10.1063/1.3566079

\section{A|P| $\begin{aligned} & \text { Journal of } \\ & \text { Applied Physics }\end{aligned}$}

Journal of Applied Physics is pleased to announce André Anders as its new Editor-in-Chief 


\title{
Perpendicular magnetic anisotropy in epitaxially strained cobalt-ferrite (001) thin films
}

\author{
H. Yanagihara, ${ }^{\text {a) }}$ Y. Utsumi, T. Niizeki, ${ }^{\text {b) }}$ J. Inoue, and Eiji Kita \\ Institute of Applied Physics, University of Tsukuba, Tsukuba 305-8573, Japan
}

(Presented 5 November 2013; received 23 September 2013; accepted 4 November 2013; published online 7 February 2014)

\begin{abstract}
We investigated the dependencies of both the magnetization characteristics and the perpendicular magnetic anisotropy of $\mathrm{Co}_{x} \mathrm{Fe}_{3-x} \mathrm{O}_{4}(001)$ epitaxial films $(x=0.5$ and 0.75$)$ on the growth conditions of the reactive magnetron sputtering process. Both saturation magnetization and the magnetic uniaxial anisotropy constant $K_{u}$ are strongly dependent on the reactive gas $\left(\mathrm{O}_{2}\right)$ flow rate, although there is little difference in the surface structures for all samples observed by reflection high-energy electron diffraction. In addition, certain dead-layer-like regions were observed in the initial stage of the film growth for all films. Our results suggest that the magnetic properties of $\mathrm{Co}_{x} \mathrm{Fe}_{3-x} \mathrm{O}_{4}$ epitaxial films are governed by the oxidation state and the film structure at the vicinity of the interface. (C) 2014 AIP Publishing LLC. [http://dx.doi.org/10.1063/1.4864048]
\end{abstract}

\section{INTRODUCTION}

Magnetic anisotropy (MA) is one of the most important properties of magnetic materials. In general, spin-orbit coupling is a primary source of origin of MA; and therefore, a large spin-orbit coupling constant and/or large orbital moments are often observed in strong MA materials. ${ }^{1}$ It has been known that bulk cobalt ferrite possesses an exclusively large cubic MA of the order of $\approx 10^{6} \mathrm{erg} / \mathrm{cm}^{3}$ among cubic spinel ferrites, and further, it exhibits remarkably large values of the magnetostriction constants. ${ }^{2}$ Thin-films of cobalt-ferrites are expected to exhibit large perpendicular magnetic anisotropy (PMA) when substrate-induced lattice distortions couple to the large magnetostriction. ${ }^{3,4}$ In the distorted films, the $\mathrm{Co}^{2+}$ ions located at the B-sites of the spinel structure probably acquire non-quenched orbital moments ${ }^{5}$ like that in the bulk. ${ }^{6-8}$ Again, MAs are mainly supposed to originate from the considerably large orbital moments of the $\mathrm{Co}^{2+}$ ions through the spin-orbit interaction. However, in cobalt-ferrite thin-films magnetic properties, such as MA and magnetization, are strongly dependent on the film-preparation methods and conditions. ${ }^{5,9-13}$ Therefore, the potential for spintronics/magnetics of the cobalt-ferrite films is unknown and even the mechanism of the PMA is not clarified yet. Thus, it is important to determine the optimum growth conditions for the cobalt ferrite films to understand the mechanism of PMA.

Although there are several reports on the magnetic properties of epitaxial cobalt-ferrite thin films grown by various different methods, such as reactive molecular beam epitaxy, ${ }^{10-12,14}$ pulsed laser deposition, ${ }^{9,13}$ and sputtering, ${ }^{15}$ most of the films grown by these methods exhibit remarkably lower saturation magnetization $\left(M_{S}\right)$ than that of the bulk material. We recently determined that reactive sputtering

\footnotetext{
${ }^{a)}$ Electronic mail: yanagiha@bk.tsukuba.ac.jp.

${ }^{b}$ Present address: Advanced Institute for Materials Research, Tohoku University, 2-1-1 Katahira, Sendai 980-8577, Japan. Electronic mail: t-niizeki@imr.tohoku.ac.jp.
}

appears to be an appropriate method to prepare spinel-type iron oxide $\left(\mathrm{Fe}_{3-\delta} \mathrm{O}_{4}\right)$ films such as $\mathrm{Fe}_{3} \mathrm{O}_{4}(001)$ and $\gamma-\mathrm{Fe}_{2} \mathrm{O}_{3}(001)$ grown on $\mathrm{MgO}(001)$ substrates. The $M_{S}$ values of the $\mathrm{Fe}_{3} \mathrm{O}_{4}(001)$ and $\gamma-\mathrm{Fe}_{2} \mathrm{O}_{3}(001)$ films are comparable with that of the bulk material. Based on our previous knowledge regarding $\mathrm{Fe}_{3-\delta} \mathrm{O}_{4}$ film growth, we have also found that $\mathrm{Co}_{x} \mathrm{Fe}_{3-x} \mathrm{O}_{4}$ epitaxial films with $x=0.75$ grown on $\mathrm{MgO}(001)$ exhibit strong uniaxial PMA of the order of 10 Merg/ $/ \mathrm{cm}^{3}$ and large $M_{S}$ values comparable with that of bulk material. ${ }^{16}$

In general, optimizing the growth conditions for reactive magnetron sputtering even with a single alloy target is complicated. These conditions include (i) growth temperature (substrate temperature), (ii) sputtering power, (iii) process pressure, and (iv) oxygen-gas flow rate among others. Since the number of parameters concerning sputtering conditions is so large, the growth conditions cannot be easily optimized. The optimized growth conditions are also possibly dependent on the composition of cobalt ferrites. In this paper, we report on the magnetic properties of cobalt ferrite epitaxial films with two different compositions grown on $\mathrm{MgO}$ (001) substrates under different conditions as a function of oxygen pressure. The results of our study provided two significant findings. First, the magnetic properties of $\mathrm{Co}_{x} \mathrm{Fe}_{3-x} \mathrm{O}_{4}$ films are dependent on the oxygen pressure during the sputtering process. Second, the $\mathrm{Co}_{x} \mathrm{Fe}_{3-x} \mathrm{O}_{4}$ films initially exhibit considerably lower magnetization values even under optimal conditions.

\section{EXPERIMENTAL}

Thin films of two different composition ratios of $\mathrm{Co}_{x} \mathrm{Fe}_{3-x} \mathrm{O}_{4}(001)(x=0.5$ and 0.75$)$ were grown epitaxially on cleaved $\mathrm{MgO}(001)$ substrates via the reactive rf magnetron sputtering technique using 2-in.-diameter Co-Fe alloy targets with the desired compositions of $\mathrm{Co}_{0.17} \mathrm{Fe}_{0.83}$ and $\mathrm{Co}_{0.25} \mathrm{Fe}_{0.75}$. Prior to film growth, $\mathrm{MgO}$ (001) substrates were annealed at $400^{\circ} \mathrm{C}$ for $30 \mathrm{~min}$. The process gas used was a mixture of $\mathrm{Ar}$ and $\mathrm{O}_{2}$. The flow rate of $\mathrm{Ar}$ was fixed at $20.0 \mathrm{sccm}$ corresponding to a partial pressure of $\approx 0.5 \mathrm{~Pa}$, 
whereas that of $\mathrm{O}_{2}\left(\phi_{\mathrm{O}_{2}}\right)$ was varied from 4.0 to $10.0 \mathrm{sccm}$ as a growth parameter. Under this condition, the $\mathrm{O}_{2}$ pressure increases by $\approx 0.1 \mathrm{~Pa}$ during the sputtering process. The growth temperature was maintained at $300{ }^{\circ} \mathrm{C}$ and the rf-power of the sputtering process was set at $100 \mathrm{~W}$. The other details of the growth conditions can be found in our previous work on epitaxial $\mathrm{Fe}_{3-\delta} \mathrm{O}_{4}$ films. ${ }^{17}$ The surface structures were observed by reflection high-energy electron diffraction (RHEED) immediately after $\mathrm{Co}_{x} \mathrm{Fe}_{3-x} \mathrm{O}_{4}$ growth. The film thicknesses were determined via the $\mathrm{X}$-ray reflectivity (XRR) technique using a conventional $\theta / 2 \theta$ diffractometer. All the magnetic measurements were carried out at room temperature (RT). The magnetization was measured using a superconducting quantum interference device, SQUID-VSM (Quantum Design, MPMS) at fields up to $\pm 70 \mathrm{kOe}$. The MAs were evaluated from the magnetic torque curves measured over the range of 0-90 kOe by a torque magnetometer (Quantum Design, PPMS Tq-Mag). The typical sample dimension was less than $2.0 \times 2.0 \mathrm{~mm}^{2}$ and the samples were shaped as squares.

\section{RESULTS AND DISCUSSION}

Figure 1 shows the typical RHEED images of the $\mathrm{MgO}$ (001) substrate and 40-nm-thick $\mathrm{Co}_{x} \mathrm{Fe}_{3-x} \mathrm{O}_{4}(001)(x=0.5)$ film grown under the condition of $\phi_{\mathrm{O}_{2}}=7.0 \mathrm{sccm}$ at a substrate temperature of $300{ }^{\circ} \mathrm{C}$. Clear streaks and Kikuchi lines can be observed in both surface images, indicating that the surface is sufficiently flat and the crystalline quality of the film is good. In the image corresponding to $\mathrm{Co}_{x} \mathrm{Fe}_{3-x} \mathrm{O}_{4}$, there are additional streaks between those corresponding to $\mathrm{MgO}$ (001), indicating that the lattice unit of $\mathrm{Co}_{x} \mathrm{Fe}_{3-x} \mathrm{O}_{4}$ is approximately twice the lattice-unit size of $\mathrm{MgO}$. Here, we remark that our $\mathrm{x}$-ray-diffraction analysis revealed that the $\mathrm{Co}_{x} \mathrm{Fe}_{3-x} \mathrm{O}_{4}$ films were coherently grown on $\mathrm{MgO}$ (001) substrates with an in-plane lattice constant that was just twice that of $\mathrm{MgO} .{ }^{16}$ The crystal lattice of the $\mathrm{Co}_{x} \mathrm{Fe}_{3-x} \mathrm{O}_{4}$ films grown on $\mathrm{MgO}(001)$ is definitely strained with in-plane stretched stress.

The results of the typical magnetization measurements at RT are shown in Fig. 2. Finite remanences are observed in the $M H$-loops under the application of an out-of-plane magnetic field, while no remanence appears in the in-plane $\mathrm{MH}$ loops, indicating that the $\mathrm{Co}_{x} \mathrm{Fe}_{3-x} \mathrm{O}_{4}(001)$ films grown on $\mathrm{MgO}(001)$ are perpendicularly magnetized films. Since the $M_{S}$ values of most of the previously reported $\mathrm{Co}_{x} \mathrm{Fe}_{3-x} \mathrm{O}_{4}$ films are significantly less than that of the bulk material, the
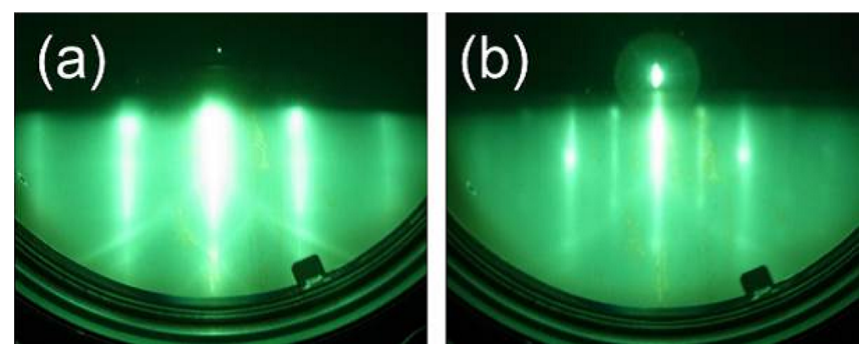

FIG. 1. RHEED images of (a) $\mathrm{MgO}(001)$, and (b) $\mathrm{Co}_{x} \mathrm{Fe}_{3-x} \mathrm{O}_{4}(x=0.5)$ samples in [100] azimuth.

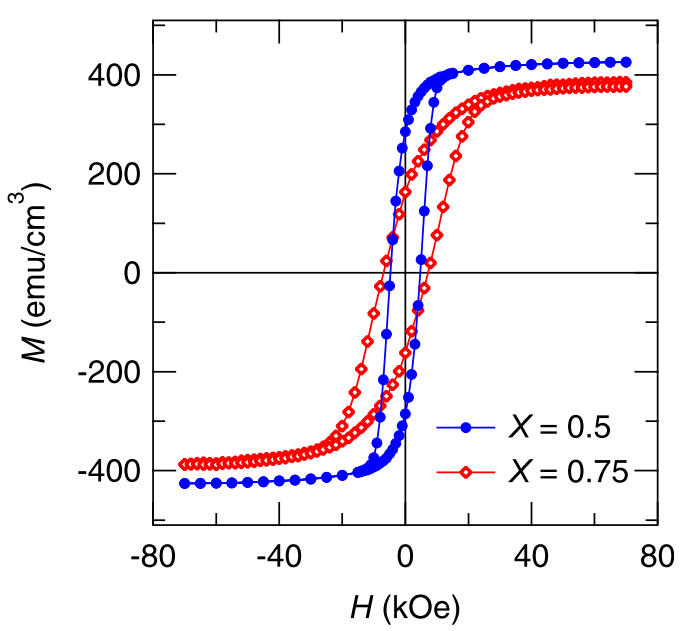

FIG. 2. Out-of-plane $M H$-loops for $\mathrm{Co}_{x} \mathrm{Fe}_{3-x} \mathrm{O}_{4}$ films with $x=0.5$ and $x=0.75$. The film thicknesses for $x=0.5$ and $x=0.75$ are $50 \mathrm{~nm}$ and $49 \mathrm{~nm}$, respectively.

$M_{S}$ value can be a good criterion to optimize the growth condition. In fact, $M_{S}$ is strongly dependent on $\phi_{\mathrm{O}_{2}}$ for all composition ratios, and some of the samples exhibit $M_{S}$ values close to the bulk value of $\mathrm{Co}_{x} \mathrm{Fe}_{3-x} \mathrm{O}_{4}$.

In order to examine the thickness dependence of the $M_{S}$ values of the $\mathrm{Co}_{x} \mathrm{Fe}_{3-x} \mathrm{O}_{4}$ films, the product of $M_{S}$ and thickness $t$ was plotted against $t$ (see Fig. 3). Each value of $M_{S} t$ was obtained by dividing the measured magnetic moment by the area of the film. The thickness values were determined by the oscillation periods observed in the XRR results. In this plot, the slopes correspond to the $M_{S}$ values, and the horizontal-axis intercept suggests the existence of a dead layer. From Fig. 3, we note that most of the $\mathrm{Co}_{x} \mathrm{Fe}_{3-x} \mathrm{O}_{4}$ films show a linear behavior, meaning that the $M_{S}$ values determined in this manner are reasonably accurate. Although the $M_{S}$ values for $x=0.75$ appears dependent on the $\phi_{\mathrm{O}_{2}}$ that for $x=0.5$ is almost constant with respect to $\phi_{\mathrm{O}_{2}}$. It is to be noted that there are finite horizontal-axis intercepts for all samples with different values of $x$ and $\phi_{\mathrm{O}_{2}}$. The typical value of the intercepts approximately ranges as $2-3 \mathrm{~nm}$, suggesting that the dead layer or the region of smaller $M_{S}$ exists in the initial growth stage. When spinel ferrite films are grown on the surfaces of non-spinel structures, such as $\mathrm{MgO}$ or $\mathrm{SrTiO}_{3}$

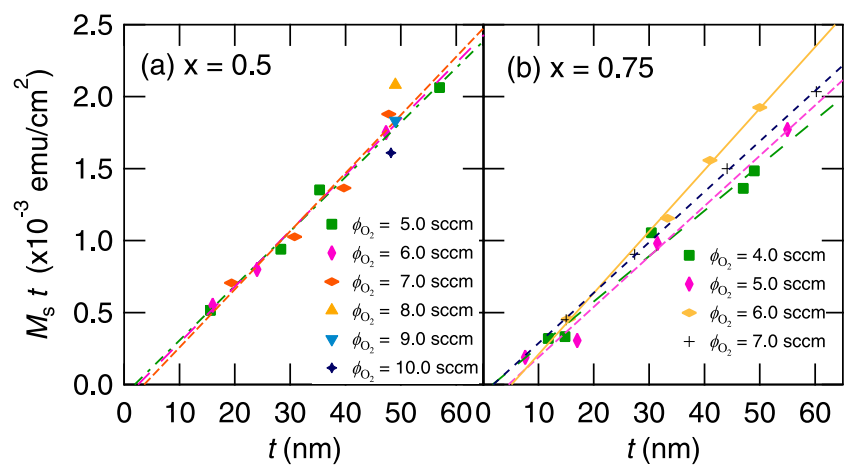

FIG. 3. Thickness dependence of $M_{S}$ for (a) $x=0.5$ and (b) 0.75 . The vertical and horizontal axes correspond to the $\mathrm{Co}_{x} \mathrm{Fe}_{3-x} \mathrm{O}_{4}$ film thickness $t$ and the product of the saturation magnetization and the thickness $\left(M_{S} t\right)$. Straight lines indicate the results of linear regression. 


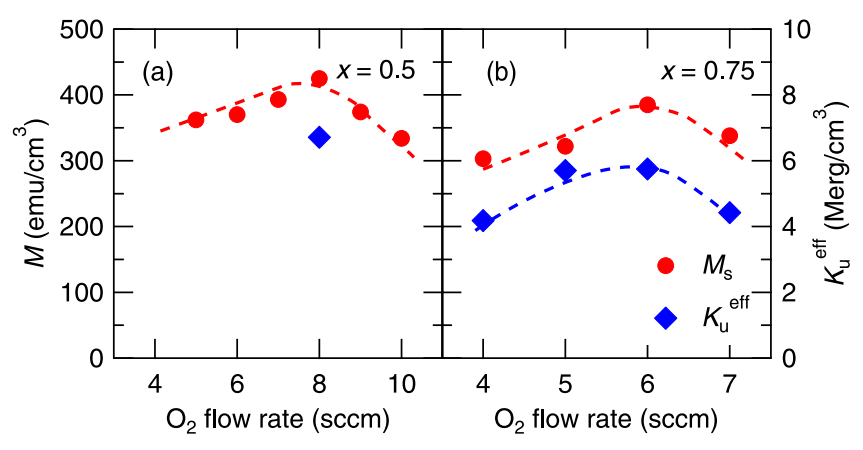

FIG. 4. Oxygen flow-rate dependence of $M_{S}$ (circles) and $K_{u}$ (diamonds) of $\mathrm{Co}_{x} \mathrm{Fe}_{3-x} \mathrm{O}_{4}$ with different composition ratios $(x=0.5,0.75)$. Broken lines serve as visual guides.

substrates, a considerable number of anti-phase boundaries (APBs) emerge, as reported for $\mathrm{Fe}_{3} \mathrm{O}_{4}$ (001) films grown on a $\mathrm{MgO}$ (001) substrate. The formation of high-density APBs results in a corresponding reduction in $M_{S}$ and/or high-field finite susceptibility. ${ }^{18}$

Figure 4 shows the $\phi_{\mathrm{O}_{2}}$ dependence of the $M_{S}$ and $K_{u}$ values of $\mathrm{Co}_{x} \mathrm{Fe}_{3-x} \mathrm{O}_{4}(001)$ for different values of $x$. For $x=0.5$, the trend of $M_{S}\left(\phi_{\mathrm{O}_{2}}\right)$ exhibits a maximum at around $\phi_{\mathrm{O}_{2}}=8.0 \mathrm{sccm}$, and the maximum $M_{S}$ is approximately $430 \mathrm{emu} / \mathrm{cm}^{3}$ that is comparable with the bulk value. On the other hand, $M_{S}\left(\phi_{\mathrm{O}_{2}}\right)$ reaches a maximum of $\approx 400 \mathrm{emu} / \mathrm{cm}^{3}$ at around $\phi_{\mathrm{O}_{2}}=6.0 \mathrm{sccm}$ for $x=0.75$. Moreover, the trend indicates that the larger- $M_{S}$ samples exhibit larger $K_{u}$.

Here, we consider how $\phi_{\mathrm{O}_{2}}$ affects the magnetic properties of $\mathrm{Co}_{x} \mathrm{Fe}_{3-x} \mathrm{O}_{4}$ films. According to our previous report on the epitaxial film growth of $\mathrm{Fe}_{3-\delta} \mathrm{O}_{4}\left(0 \leq \delta \leq \frac{1}{3}\right)$ by using the reactive magnetron sputtering technique, $\phi_{\mathrm{O}_{2}}$ directly affects the sputtering mode. ${ }^{17}$ We noticed that the presence of a threshold $\phi_{\mathrm{O}_{2}}$ value determining whether the sputtering process was the metal or oxide mode. In addition, depending on the sputtering mode, $\mathrm{Fe}_{3} \mathrm{O}_{4}$ and $\gamma-\mathrm{Fe}_{2} \mathrm{O}_{3}$ films can be selectively grown; in other words, the ratios of $\mathrm{Fe}^{2+}$ and $\mathrm{Fe}^{3+}$ ions are controlled by $\phi_{\mathrm{O}_{2}}$. Similar to the case of $\mathrm{Fe}_{3-\delta} \mathrm{O}_{4}$ films, the valences of both $\mathrm{Fe}$ and $\mathrm{Co}$ ions in the $\mathrm{Co}_{x} \mathrm{Fe}_{3-x} \mathrm{O}_{4}$ films may possibly undergo changes via variation of $\phi_{\mathrm{O}_{2}}$, resulting in the observed $M_{S}$ values of $\mathrm{Co}_{x} \mathrm{Fe}_{3-x} \mathrm{O}_{4}$ films.

Finally, we briefly comment on the relation between $M_{S}$ and $x$. As shown above, $M_{S}$ equals the saturation magnetization value of bulk $\mathrm{Co}_{x} \mathrm{Fe}_{3-x} \mathrm{O}_{4}$ for $x=0.5$ and 0.75 upon carefully tuning the $\phi_{\mathrm{O}_{2}}$. Although experimental results for $\mathrm{Co}_{x} \mathrm{Fe}_{3-x} \mathrm{O}_{4}$ films with $x=1$ are not presented in this paper, it appears fairly difficult to prepare a $\mathrm{CoFe}_{2} \mathrm{O}_{4}$ film with an $M_{S}$ comparable with that of the bulk material in our attempts. Assuming that the valencies of $\mathrm{Fe}$ and $\mathrm{Co}$ are $3+$ and $2+$, respectively, for the sample corresponding to $x=1$ and that the film also possesses an inverse spinel structure, the possible combinations of the B-site occupation by the same number of $\mathrm{Fe}^{3+}$ and $\mathrm{Co}^{2+}$ ions is limited, in the same manner as Anderson's criterion for Verwey ordering in $\mathrm{Fe}_{3} \mathrm{O}_{4}{ }^{19}$ Therefore, it could be difficult for the $\mathrm{Fe}^{3+}$ and $\mathrm{Co}^{2+}$ ions to migrate between the B-sites and occupy optimized positions within a limited interval of time. As a result the samples with $x \approx 1$ possibly contain a large number of various defects. Although this is no more than a speculation, in order to grow the $\mathrm{Co}_{x} \mathrm{Fe}_{3-x} \mathrm{O}_{4}$ films with $x=1$ by the sputtering technique, we speculate that tuning of the other parameters pertaining to the growth conditions may be required.

\section{SUMMARY}

We investigated the dependencies of both the magnetization characteristics and the perpendicular magnetic anisotropy of $\mathrm{Co}_{x} \mathrm{Fe}_{3-x} \mathrm{O}_{4}(001)$ epitaxial films $(x=0.5$ and 0.75$)$ on the $\mathrm{O}_{2}$ flow rate in the reactive magnetron sputtering process. The thickness-dependent magnetization measurements revealed the existence of dead-layer regions in the initial stage of the film growth for all films. Our results suggest that the magnetic properties of $\mathrm{Co}_{x} \mathrm{Fe}_{3-x} \mathrm{O}_{4}$ epitaxial films are governed by the oxidation state and the film structure during initial growth.

\section{ACKNOWLEDGMENTS}

This work was supported by the Elements Science and Technology Project of MEXT, Japan. The $M H$-loop measurements were carried out at NIMS, Tsukuba.

${ }^{1}$ S. Chikazumi, Physics of Ferromagnetism (Wiley, 1964).

${ }^{2}$ R. Bozorth, E. Tilden, and A. Williams, Phys. Rev. 99, 1788 (1955).

${ }^{3}$ A. Lisfi, C. M. Williams, L. T. Nguyen, J. C. Lodder, A. Coleman, H. Corcoran, A. Johnson, P. Chang, A. Kumar, and W. Morgan, Phys. Rev. B 76, 54405 (2007).

${ }^{4}$ J. Inoue, H. Itoh, M. A. Tanaka, K. Mibu, T. Niizeki, H. Yanagihara, and E. Kita, IEEE Trans. Magn. 49, 3269 (2013).

${ }^{5}$ J. A. Moyer, C. A. F. Vaz, D. P. Kumah, D. A. Arena, and V. E. Henrich, Phys. Rev. B 86, 174404 (2012).

${ }^{6}$ K. Yosida and M. Tachiki, Prog. Theor. Phys. 17, 331 (1957).

${ }^{7}$ J. Slonczewski, Phys. Rev. 110, 1341 (1958).

${ }^{8}$ J. C. Slonczewski, J. Appl. Phys. 32, S253 (1961).

${ }^{9}$ P. C. Dorsey, P. Lubitz, D. B. Chrisey, and J. S. Horwitz, J. Appl. Phys. 79, 6338 (1996).

${ }^{10}$ S. Chambers, R. Farrow, S. Maat, M. Toney, L. Folks, J. Catalano, T. Trainor, and G. Brown, J. Magn. Magn. Mater. 246, 124 (2002).

${ }^{11}$ A. V. Ramos, S. Matzen, J.-B. Moussy, F. Ott, and M. Viret, Phys. Rev. B 79, 14401 (2009).

${ }^{12}$ W. Huang, J. Zhu, H. Z. Zeng, X. H. Wei, Y. Zhang, and Y. R. Li, Appl. Phys. Lett. 89, 262506 (2006).

${ }^{13}$ Y. Suzuki, R. B. van Dover, E. M. Gyorgy, J. M. Phillips, V. Korenivski, D. J. Werder, C. H. Chen, R. J. Cava, J. J. Krajewski, J. W. F. Peck, and K. B. Do, Appl. Phys. Lett. 68, 714 (1996).

${ }^{14}$ H. Yanagihara, K. Uwabo, M. Minagawa, E. Kita, and N. Hirota, J. Appl. Phys. 109, 07C122 (2011).

${ }^{15}$ C. Jin, H. Liu, P. Li, D. F. Kuang, and H. L. Bai, J. Appl. Phys. 110, 013917 (2011).

${ }^{16}$ T. Niizeki, Y. Utsumi, R. Aoyama, H. Yanagihara, J.-i. Inoue, Y. Yamasaki, H. Nakao, K. Koike, and E. Kita, Appl. Phys. Lett. 103, 162407 (2013).

${ }^{17}$ H. Yanagihara, M. Myoka, D. Isaka, T. Niizeki, K. Mibu, and E. Kita, J. Phys. D: Appl. Phys. 46, 175004 (2013).

${ }^{18}$ D. Margulies, F. Parker, M. Rudee, F. Spada, J. Chapman, P. Aitchison, and A. Berkowitz, Phys. Rev. Lett. 79, 5162 (1997).

${ }^{19}$ P. W. Anderson, Phys. Rev. 102, 1008 (1956). 